\title{
Staphylococci phages display vast genomic diversity and evolutionary relationships
}

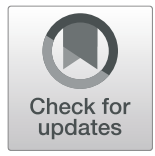

\author{
Hugo Oliveira ${ }^{1 *}$ (D), Marta Sampaio ${ }^{1}$, Luís D. R. Melo ${ }^{1}$, Oscar Dias ${ }^{1}$, Welkin H. Pope ${ }^{2}$, Graham F. Hatfull² and \\ Joana Azeredo ${ }^{1}$
}

\begin{abstract}
Background: Bacteriophages are the most abundant and diverse entities in the biosphere, and this diversity is driven by constant predator-prey evolutionary dynamics and horizontal gene transfer. Phage genome sequences are under-sampled and therefore present an untapped and uncharacterized source of genetic diversity, typically characterized by highly mosaic genomes and no universal genes. To better understand the diversity and relationships among phages infecting human pathogens, we have analysed the complete genome sequences of 205 phages of Staphylococcus sp.

Results: These are predicted to encode 20,579 proteins, which can be sorted into 2139 phamilies (phams) of related sequences; 745 of these are orphams and possess only a single gene. Based on shared gene content, these phages were grouped into four clusters (A, B, C and D), 27 subclusters (A1-A2, B1-B17, C1-C6 and D1-D2) and one singleton. However, the genomes have mosaic architectures and individual genes with common ancestors are positioned in distinct genomic contexts in different clusters. The staphylococcal Cluster B siphoviridae are predicted to be temperate, and the integration cassettes are often closely-linked to genes implicated in bacterial virulence determinants. There are four unusual endolysin organization strategies found in Staphylococcus phage genomes, with endolysins predicted to be encoded as single genes, two genes spliced, two genes adjacent and as a single gene with inter-lytic-domain secondary translational start site. Comparison of the endolysins reveals multi-domain modularity, with conservation of the SH3 cell wall binding domain.
\end{abstract}

Conclusions: This study provides a high-resolution view of staphylococcal viral genetic diversity, and insights into their gene flux patterns within and across different phage groups (cluster and subclusters) providing insights into their evolution.

Keywords: Staphylococcus, Bacteriophages, Genomes, Clusters, Phams, Endolysin

\section{Background}

Bacteriophages (phages) - viruses of bacteria - are ubiquitous, and are the most populous (over $10^{31}$ ) and diverse of all biological entities [1,2]. Phage predation affects not only the microbial balance $[3,4]$, but also food webs [5], biogeochemical cycles [6] and human diseases [7]. Phages are able to kill 50\% of the bacteria produced every $48 \mathrm{~h}$, playing a major role in microbial ecology and in the evolution of bacterial genomic structures through horizontal gene transfer (HGT), including virulence factors [8].

\footnotetext{
* Correspondence: hugooliveira@deb.uminho.pt

${ }^{1}$ CEB - Centre of Biological Engineering, University of Minho, Braga, Portugal Full list of author information is available at the end of the article
}

Up to January 2019, there have been 5595 complete Caudovirales genome sequences recorded in the RefSeq database at GenBank. The Caudovirales (tailed phages with dsDNA), are the most commonly isolated viruses. Phages of phylogenetically distant hosts, and often from the same host, typically share little or no DNA sequence similarity, and no universal genes [9], confounding their taxonomic classification. While nucleotide sequencebased methods such as pairwise genome alignment using BLASTN, average nucleotide identity (ANI), or dot plot analysis are useful for studying closely-related phages, analyses using shared gene content based on protein sequence similarity enlighten more distant relationships, and illustrate the diversity continuum in viral sequence space $[10,11]$. These studies were undertaken for phages of

(c) The Author(s). 2019 Open Access This article is distributed under the terms of the Creative Commons Attribution 4.0 International License (http://creativecommons.org/licenses/by/4.0/), which permits unrestricted use, distribution, and reproduction in any medium, provided you give appropriate credit to the original author(s) and the source, provide a link to the Creative Commons license, and indicate if changes were made. The Creative Commons Public Domain Dedication waiver (http://creativecommons.org/publicdomain/zero/1.0/) applies to the data made available in this article, unless otherwise stated. 
Mycobacterium sp. $(n=627)$ [12], Enterobacteria $(n=337)$ [13], Bacillus sp. $(n=93)$ [14], Gordonia sp. $(n=79)$ [10] and Arthrobacter sp. $(n=46)$ hosts [15]. Mycobacterium phages represent the largest group of phages infecting a single host, Mycobacterium smegmatis $\mathrm{mc}^{2} 155$; and early studies highlighted their high genetic diversity and genome mosaicism [16, 17]. A recent study analysed over 700 genomes of Actinobacteria phages that could be sorted into 30 distinct phage clusters [10]. The Enterobacteria phages, isolated by several investigators on multiple hosts, were sorted into 56 clusters; phage of Bacillus sp., Gordonia sp. and Arthrobacter sp., were likewise sorted into related groups $[10,14,15]$. Although these surveys included hosts of different taxonomic levels, there is an evident genetic phage diversity that often includes genomes with mosaic architectures and genes of unknown function which lack homology [18].

A previous study compared the genomes of 85 Staphylococcus phages, mostly isolated from S. aureus host, and grouped them into three classes (Class I, Class II and Class III) based on their genome size, gene order, and nucleotide and protein sequences [19]. Here, we have extended the comparative genomic analysis to 205 phages infecting several species of staphylococci. We comparatively analyzed the genomes at the nucleotide and proteomic level and used a 35\% shared gene content cut-off to place phages solely in one cluster. These phages, which were isolated at various times and from different environments, provide a high-resolution view of the genetic diversity among all members infecting these clinical relevant pathogens.

\section{Results}

Staphylococcal phages can be grouped in four clusters, 27 subclusters and one singleton

To determine the relationship of staphylococci phages, all complete genomes sequences deposited at GenBank as of October 2018 were retrieved and analysed using ANI, shared gene content and gene content dissimilarity metrics as recently described [10]. BLASTN and average nucleotide identity to identify whole phage genomes and genome regions with nucleotide sequence similarity and Phamerator to generate protein phamilies (phams) for calculating pairwise shared gene content and genome architecture. The dataset includes 205 genomes ranging from $16.8 \mathrm{~kb}$ (phage 44AHJD) to $151.6 \mathrm{~kb}$ (phage vB_SauM_0414_108) in size, coding between 20 to 249 predicted genes, and isolated from eleven different hosts, including nine coagulase-negative and three coagulasepositive or variable species (Additional file 1).

Comparative analysis of all 205 staphylococcal phage genomes identified 20,579 predicted proteins, which were sorted into 2139 phamilies (phams) of related sequences, 745 of which possess only a single sequence (orphams)
(Additional file 2). Based on average shared gene content as determined by pham membership, these phages can be grouped into four clusters (A-D), 27 subclusters (A1-A2, B1-B17, C1-C6 and D1-D2) and one singleton (with no close relatives) (Fig. 1). A threshold value of 35\% average pairwise shared gene content was used to cluster genomes, as described for Gordonia and Mycobacterium phages [10, 12]. These groupings are supported by pairwise ANI values (Additional file 3) and gene content similarity (Additional file 4). Cluster members exhibit similar virion morphology and genometrics (size, number of ORF and GC content) (Additional file 1). To further analyse relationships, we defined conserved (phams found in all phages), accessory (phams present in at least three phages) and unique (orphams, present in only one phage) phams amongst members of each cluster/subclusters, providing further insights into specific gene pattern exchanges (Additional file 5). Specific examples are provided below.

\section{Cluster $A$}

The sixteen Cluster A staphylococci phages are morphologically podoviral and can be divided into two subclusters (A1, A2). Cluster A phages are an extremely well-conserved group with respect to nucleotide and amino acid homology, morphology, lytic lifestyle, genome size (16-18 kb), GC content (27-29\%), and predicted number of genes (20 to 22) (Additional file 1). The genomes are organized into left and right arms, with rightwards- and leftwards-transcription in the left and right arms, respectively (Additional files 6, 7). Interestingly, the DNA packaging and DNA polymerase genes are located near the start of the left genome terminus, with the other structural protein genes located in the right arm [20]. Subcluster A1 has 14 phages (e.g. BP39, GRCS) that share substantial ANI (> 86\%) and gene content $(>82 \%$ ) (Additional file 6), but differ in arrangements of the tail fiber genes (44AHJD, SLPW and 66). Subcluster A2 includes two phages (St134 and Andhra), that infect $S$. epidermidis (Additional file 7). These phages have high ANI (92\%) and shared gene content (98\%) values. Subcluster A1 and A2 phages vary in a tail endopeptidase gene upstream of the DNA encapsulation protein. Overall, the high number of conserved phams (17 to 20) and limited number of accessory phams ( 1 to 5 ) or unique phams (1 to 2 ) reflects the genomic homogeneity of Cluster A phages (Additional file 5). About $60 \%$ of genes have predicted functions related to DNA replication (DNA binding, DNA polymerase), virion morphology (DNA packaging, tail fiber, collar and major capsid) or cell lysis (holin and endolysin) (Additional file 2).

\section{Cluster B}

Cluster B is the largest and most diverse cluster, with 132 phage isolates from multiple different hosts $(S$. aureus, $S$. epidermidis, S. pseudintermedius, S. sciuri, S. haemolyticus, 


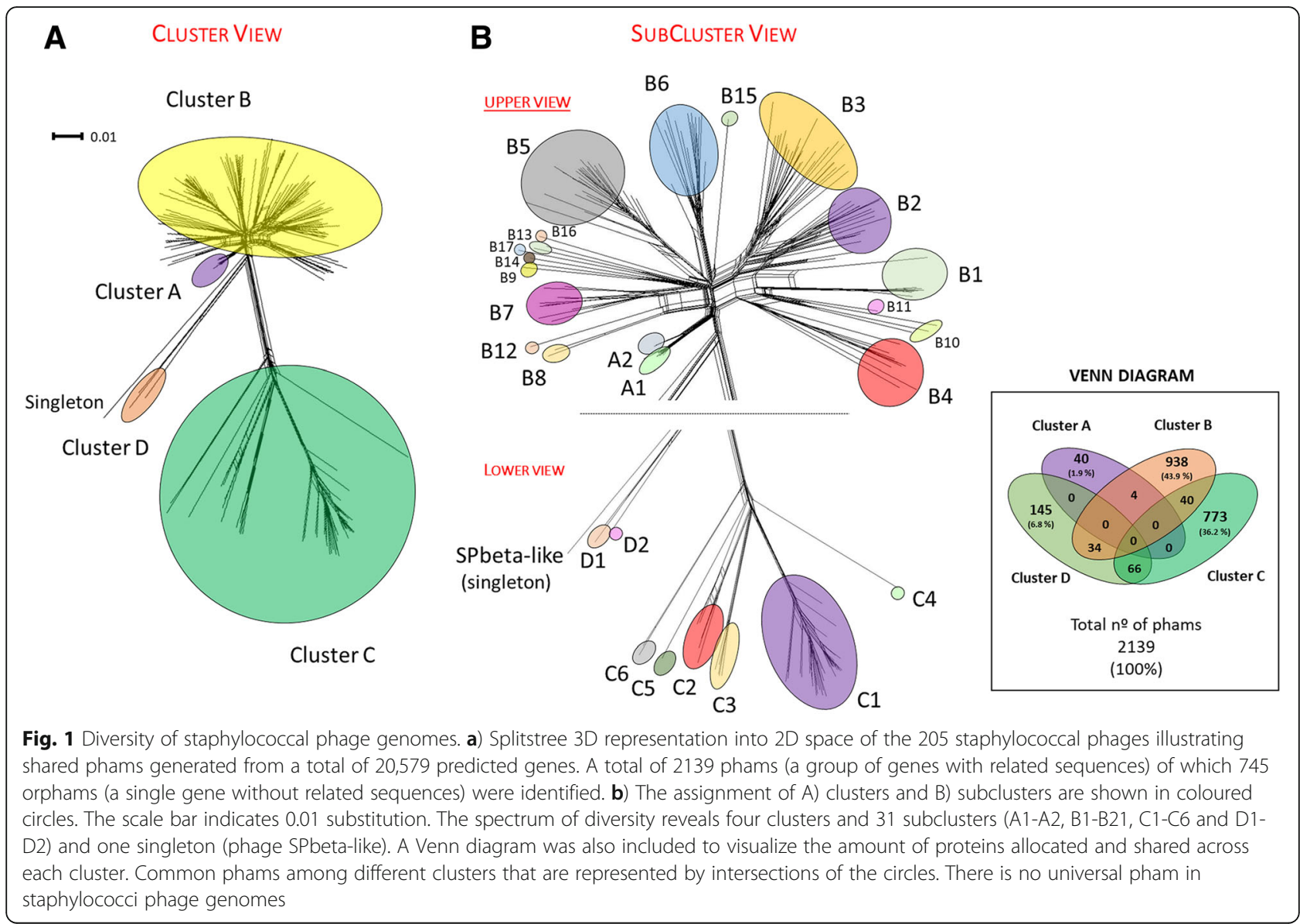

S. saprophyticus, S. capitis and S. warneri). Most are predicted to be temperate and the genome sizes vary from 39.6 to $47.8 \mathrm{~kb}$ with $42-79$ predicted protein-encoding genes. The genomes are organized into a rightwards-transcribed left arm containing structural genes and the lysis cassette, a central leftwards-transcribed integration cassette, and a rightwards-transcribed right arm coding for many small proteins of unknown functions (Additional files 8, 9, $10,11,12,13,14,15,16,17,18,19,20,21,22,23,24)$. Cluster $B$ phages are divided into 17 subclusters based on manual inspection of gene content similarity, genome pairwise comparisons, and ANI values (Additional files 8, 9, 10, 11, 12 , 13, 14, 15, 16, 17, 18, 19, 20, 21, 22, 23, 24, Additional files 3,4$)$. The larger subclusters are $\mathrm{B} 1(n=7), \mathrm{B} 2(n=19)$, B3 $(n=26)$, B4 $(n=9)$, B5 $(n=26)$, B6 $(n=18)$ and B7 $(n=$ 12 ) and have phages with collinear genomes (Additional file $8,9,10,11,12,13,14)$. While subclusters B1-B2 and B3-B7 were exclusively isolated from $S$. pseudintermedius or $S$. aureus hosts, B4 is unusual in having phages isolated from S. aureus, S. haemolyticus and S. epidermidis (Additional file 1). The remaining B8-B17 subclusters each contain only three or fewer members, mostly isolated from rarer coagulase-negative hosts, such as $S$. sciuri, $S$ warneri, $S$ saprophyticus S. haemolyticus and S. hominis. Although they have similar genome organizations to other Cluster B phages, fewer than $42 \%$ of their genes are shared with them (Additional file 15, 16, 17, 18, 19, 20, 21, 22, 23, 24).

Cluster B phages are predicted to be temperate, and encode predicted integrase and repressor genes; prophage establishment had been demonstrated for phages phiPV83, phiNM1, phiNM2, phiNM4, vB_SepiS-philPLA5, vB_SepiS-phiIPLA7, 11, 42E, phi12 and phi13 [21-24]. Generally, in Cluster B genomes, about $40-50 \%$ of the predicted genes are functionally annotated with roles of DNA packaging, virion structure, cell lysis, lysogeny, or DNA replication. Overall, the spectrum of diversity of this large Cluster B is high and although all members are related through gene content similarity to at least one of the phages (>35\%), some viruses (e.g. IME1367_01, IME-SA4, phiRS7, StB20, StB20-like) have lower pairwise shared gene content $(<35 \%)$. Subcluster B1 is by far the most conserved B subcluster, with members sharing 46 conserved phams, while subcluster B2 and B4 are the most heterogeneous groups with only ten or fewer conserved phams (Additional file 5). Less than 50\% protein-encoding genes have known functions in the Cluster B phages. 


\section{Cluster C}

The 53 Cluster $C$ phages are morphologically members of Myoviridae, with genome sizes ranging from $127.2 \mathrm{~kb}$ to $151.6 \mathrm{~kb}$ coding for $164-249$ predicted proteins. Cluster $\mathrm{C}$ can be divided into six subclusters. Cluster $\mathrm{C} 1$ phage genomes are characterized by direct terminal repeats, base pair 1 of these genomes is selected to be the first base of the repeat; for other Cluster $C$ phages base pair 1 is identified as the first base of the terminase gene (as per convention). Most genes are transcribedrightwards, with the rightmost $20 \mathrm{~kb}$ transcribed leftwards (Additional files 25, 26, 27, 28, 29, 30). While the variation in predicted gene content is due in part to small insertion/deletions, some (10\%) arise from inconsistencies in the annotations.

Subcluster C1 $(n=37)$ is the most numerous Cluster C subcluster comprised of $S$. aureus infecting phages (e.g. K and P108) (Additional file 25), and are well-conserved with ANIs $>71 \%$ and shared gene content $>72 \%$ (Additional files 3,4$)$. Cluster $\mathrm{C} 1$ phages have direct terminal repeats of $\sim 8 \mathrm{~kb}$, suggesting a common dsDNA packaging mechanism (Additional file 1). This subcluster is composed of phages described to have broad-host range (e.g. K) and with therapeutic potential [25].

Subcluster C2 $(n=6)$ has closely related $S$. aureus-infecting phages (Stau2, StAP1, vB_SauM_Remus, vB_SauM_Romulus, SA11 and qdsa001), with high ANI $(>95 \%)$ and shared gene content $(>77 \%)$ values (Additional file 26). They encode between 164 to 199 genes; Stau2 and Sa11 are the only members known to encode RNA ligase. The remaining phages are distributed between subclusters $\mathrm{C} 3$ ( $n=5$, phiIPLA-C1C, phiIBB-SEP1, Terranova, Quidividi and Twillingate), C4 (Twort), C5 (vB_SscM-1 and vB_SscM-2) and C6 (phiSA_BS1 and phiSA_BS2), respectively (Additional files 27, 28, 29, 30). All members of subclusters $\mathrm{C} 3, \mathrm{C} 4$ and $\mathrm{C} 5$ share fewer than $60 \%$ of their genes with other phages of Cluster $C$; these phages, such as Twort, are known to infect rare serotypes of host species that share limited nucleotide identity to S. aureus. Overall, all Cluster $C$ phages have a relatively high number of shared phams (Additional file 5), but fewer than $40 \%$ of their genes have predicted functions.

\section{Cluster D}

Cluster D is comprised of three lytic Siphoviridae, 6ec, vB_SepS_SEP9 and vB_StaM_SA2, with genome sizes ranging from $\sim 89-93 \mathrm{~kb}$, coding for $129-142$ predicted proteins. The genomes have defined cohesive termini with 10 base 3' single stranded DNA extensions (Additional file 1) [26]. The left arms are rightwards-transcribed and code for virion proteins, cell lysis functions (holin and endolysin) and predicted general recombinases (Additional files 31, 32). The right arms are leftwards-transcribed, with a leftwards-transcribed five $\mathrm{kb}$ insertion near the right genome end (Additional files 31, 32). The right arm contains genes with predicted functions in DNA replication (e.g. DNA polymerase) and DNA metabolism (e.g. ribonucleotide reductase) genes. The two short rightmost operons code for small proteins of unknown function. Cluster D phages do not have predicted lysogeny functions, although they code for a tyrosine recombinase in the left arm (pham 1333); a similar arrangement has been identified in lytic Gordonia phages [10]. It is unclear what specific role these recombinases play. Morphologically, phages 6ec and SEP9 have very long flexible tails $(>300 \mathrm{~nm})$, twice as long as those of Cluster B phages [26, 27]. We also note that phage vB_SepS_SEP9 has relatively high $\mathrm{G}+\mathrm{C}$ content of 45.8 , $10 \%$ higher than the other staphylococcal phages (Additional file 1). This may reflect either a broader host range than other staphylococcal phages, or be a consequence of its recent evolutionary history [27].

Cluster D is subdivided into two subclusters based on ANI. Subcluster D1 has two members (6ec, vB_SepS_SEP9) with high ANI (78\%) and shared gene content (77\%) values and are organized collinearly (Additional file 31). Subcluster D2 has a single member (vB_StaM_SA2), which shares $45 \%$ or fewer genes with the subcluster D1 phages (Additional file 32). Although not yet examined by electron microscopy, vB_StaM_SA2 is predicted to have a similarly long noncontractile tail found in subcluster D1 members due to the similarity between the tail proteins, particularly the tape measure proteins (see pham 814 of Additional file 2). Cluster D phages have functions assigned only to about $35 \%$ of the predicted genes.

\section{Phage SPbeta-like}

The singleton phage SPbeta-like is a siphovirus sharing fewer than $10 \%$ of its genes with other staphylococcal phages (Additional file 33). SPbeta-like has a genome of $127,726 \mathrm{bp}$ and encodes 177 genes organized into three major operons, of which only $30 \%$ have predicted functions; these include virion proteins (e.g. tape measures protein), cell lysis (holin and endolysin), DNA replication (e.g. DNA polymerase and helicase), and three predicted recombinases (phams 139, 415, 1023). Similarly to Cluster D phages, SPbeta-like lacks genes associated with stable maintenance of lysogeny.

\section{Gene content reflects the diversity of Staphylococcus phages}

To further assess diversity of Staphylococcus phages and clusters, we calculated pairwise gene content dissimilarity (GCD) and maximum GCD gap distance (MaxGCDGap) metrics (Fig. 2a-f), as described previously [10, 11]. The GCD metric ranges from 1 (no shared 0 genes) to 0 (all genes are shared). We generated three datasets, the first including Staphylococcus sp. phages $(n=205)$, the 


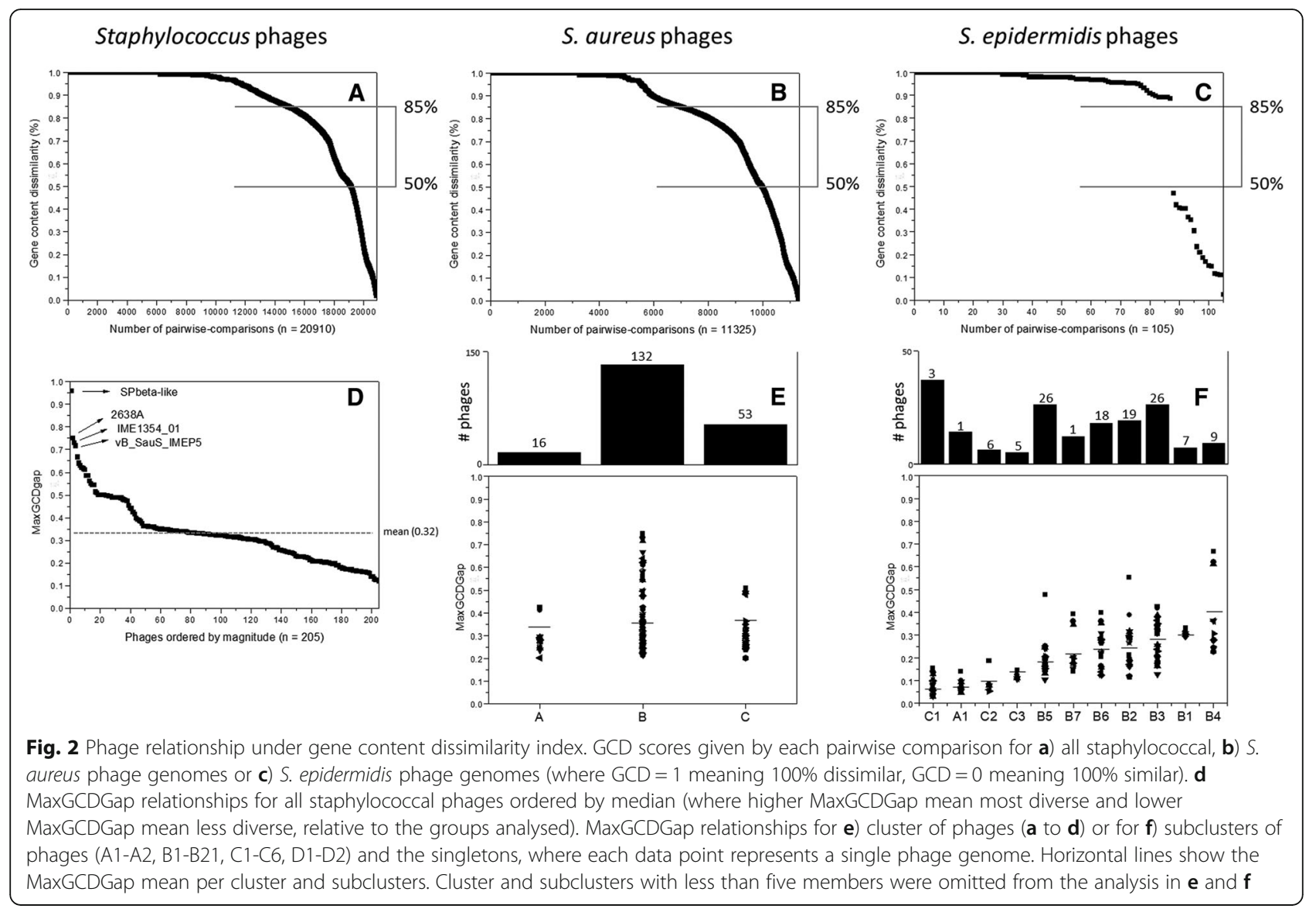

second with only those isolated on $S$. aureus $(n=162)$, and the third including S. epidermidis phages $(\mathrm{n}=16)$ (Fig. 2a-c). Of 20,910 staphylococcal phage pairwise comparisons, the majority (78\%) share $20 \%$ or fewer genes (GCD >0.8), (Fig. 2a); likewise, of $11,325 \mathrm{~S}$. aureus phage pairwise comparisons, $71 \%$ had $20 \%$ or fewer shared genes (GCD > 0.8) (Fig. 2b). However, within the 105 S. epidermidis phage pairwise comparisons, $83 \%$ had $20 \%$ or fewer shared genes (GCD $>0.8$ ), (Fig. 2c). Staphylococcus sp. and S. aureus-infecting phages exhibited a number of pairwise comparisons $(225 \%)$ that yielded GCD values between 0.85 and 0.50 , reflecting between 15 and $50 \%$ shared genes, respectively. None of the $S$. epidermidis phage pairwise comparisons were found in this range, indicating that the S. epidermidis phages primarily shared phams with closely related phages, and not with unrelated phages.

Rank ordered GCD pairwise comparisons illustrate the continuum of diversity found in any particular set of phages with sufficient members; the largest difference between two adjacent points is termed MaxGCDGap. Phages in datasets with a large MaxGCDGap exhibit cluster isolation, with fewer phages sharing phams with non-cluster members. MaxGCDGap can range from near 0 (indicating small gene content discontinuities, all phages are closely related) to 1 (indicating large gene content discontinuities, no phages are closely related). Although this metric is dependent on the dataset size and composition, the spectrum of genetic diversity can be further resolved with additional genomes [10]. With the exception of SPbeta-like, MaxGCDGap values show an almost uninterrupted spectrum from 0.75 to 0.12 , with a mean value of 0.33 (Fig. 2d), the singleton SPbeta-like has a much higher MaxGCDGap value of 0.96 , as expected. We also plotted MaxGCDGap values ordered by magnitude per cluster and per subcluster (Fig. 2e-f), showing a broad range of values, reflecting the spectrum of diversity in the entire phage genome set. We noted a lower variability of MaxGCDGap in clusters $A$ and $C$, indicative of that they are well-conserved groups, in comparison with Cluster B (and in particular subcluster B4), that possess broader range and higher MaxGCDGap values reflecting a greater diversity. Similar observations of different levels of gene content discontinuities have been described previously, with Propionibacterium or Arthrobacter phages and Mycobacteria or Synechococcus phages, as examples of good and poorly conserved groups, respectively [10]. 


\section{Staphylococci phages display multiple integration systems}

Temperate phages have the ability to integrate into the bacterial chromosome and reside as prophages. As the unidirectional site-specific integration of phage genome into bacterial chromosome is mediated by integrases, we analysed relationships between the integrase types and Cluster B phages $(n=132)$ that are either temperate or virulent-derivatives of temperate phages; many have been identified as prophages in bacterial genomes (e.g. phi13, phiNM1, phiNM2, phiNM3 and phiNM4) (Fig. 3 and Additional file 34) [21, 28]. We identified integrases in two distinct groups that used either tyrosine or serine as catalytic residues: tyrosine (Y-Int) and serine recombinases (S-Int). Almost all Cluster B staphylococci phages have predicted integrases with the exception of $3 \mathrm{~A}$ and StB20-like, which likely lost them due to recombination and deletion. The integrases were assigned to five phams; all the serine integrases are members of the same pham, and the tyrosine integrases into the remaining four phams (Fig. 3, Table 1). All of the tyrosine integrases possess a single shared pfam domain (phage_integrase domain, pfam00589), while the S-Int have a different pfam domain in common (C-terminal recombinase, pfam07508). Although Goerke et al. have previously attempted to classify phages according to phage integrases obtaining seven major and eight minor groups [29], our updated dataset demonstrated that no obvious link between type of integrase, host species or subcluster could be made; the same integrase can be detected within phages within different B subclusters and in phages with different hosts. For example, a member of pham 148, which contains the most members within the integrase phams is found in at least one phage from each of the B subclusters, excepting only B1, B11 and B13 (Table 1). The pham with the fewest members, 1656, is found only within a phage in the B8 subcluster, although, other B8 subcluster members contain integrases

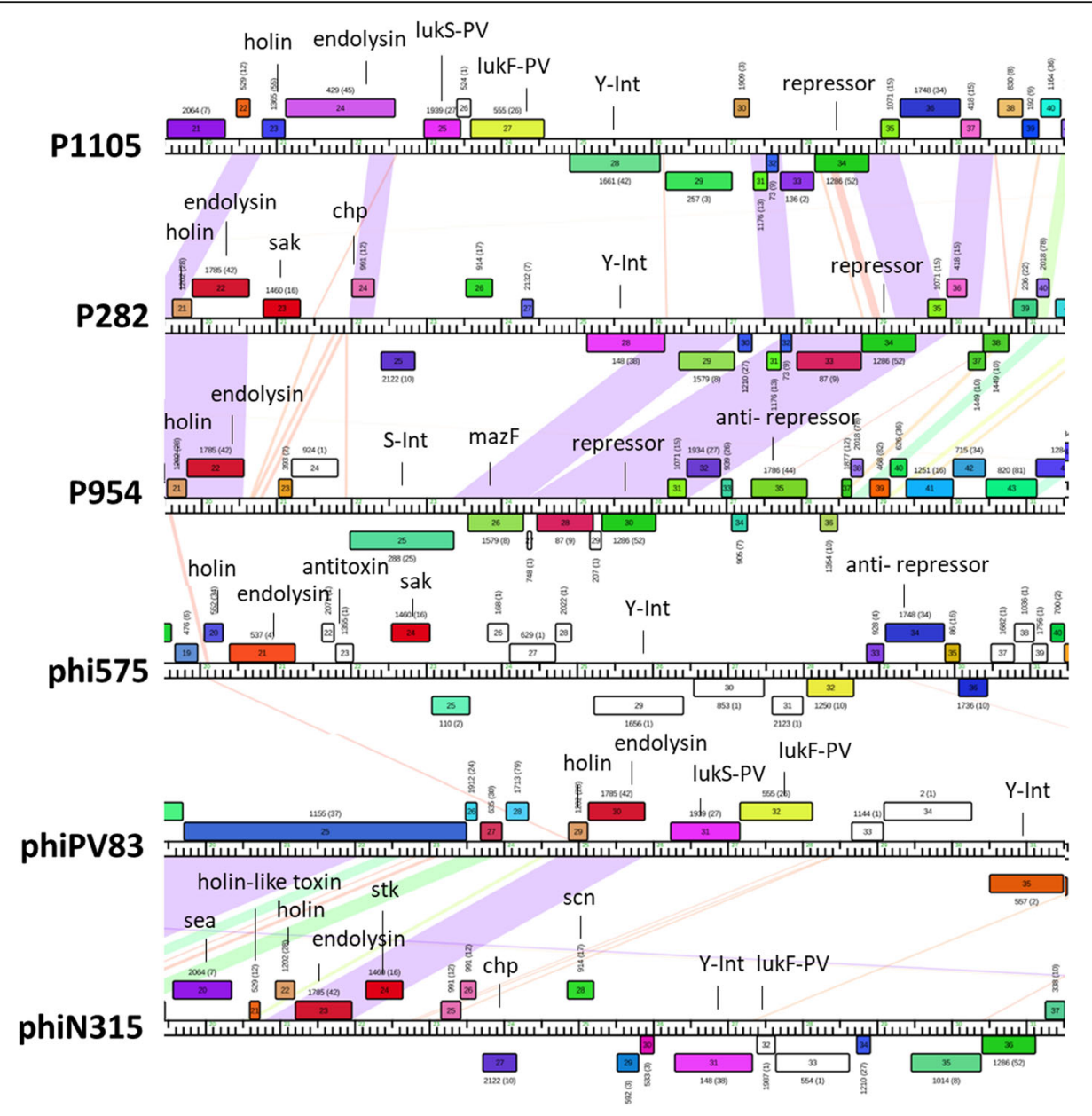

Fig. 3 Diversity of staphylococcal phage integrases. Maps of the lysis cassettes, virulence determinants, and integration cassettes for six Staphylococcus phages were constructed using Phamerator, genes are labelled with their putative functions where applicable 
Table 1 Staphylococcal cluster B phage integrases. The dataset includes 205 staphylococcal phages, of which 132 belong to the cluster B Siphoviridae. Phams related to integration functions and virulence determinants are represented to phage member, clusters and protein domains

\begin{tabular}{|c|c|c|c|c|c|}
\hline Pham & Function & Alternative nomenclature $^{a}$ & Number of members & Domains $^{b}$ & Conserved, accessory or unique pham \\
\hline \multicolumn{6}{|c|}{ Integrases } \\
\hline 148 & Y-Int & Sa3, Sa9, Sa10, Sa11 & 38 & pfam14659; pfam00589 & $\begin{array}{l}\text { Conserved (B9); Accessory } \\
\text { (B2, B3, B4, B5, B6, B7, B10); } \\
\text { Unique (B8, B12, B14, B15, B16, B17) }\end{array}$ \\
\hline 280 & Y-Int & Sa1, Sa5 & 27 & $\begin{array}{l}\text { pfam14657; pfam14659; } \\
\text { pfam00589 }\end{array}$ & $\begin{array}{l}\text { Conserved (B1); Unique (B7); } \\
\text { Accessory (B2, B3) }\end{array}$ \\
\hline 288 & S-Int & Sa7, Se1, Se12 & 25 & pfam00239; pfam07508 & $\begin{array}{l}\text { Accessory (B2, B3, B4); Unique } \\
\text { (B6, B10, B11, B13) }\end{array}$ \\
\hline 1656 & Y-Int & - & 1 & pfam14659; pfam00589 & Unique (B8) \\
\hline 1661 & Y-Int & Sa2, Sa6 & 40 & pfam00589 & Accessory (B3, B5, B6, B7) \\
\hline \multicolumn{6}{|c|}{ Virulence determinants } \\
\hline 297 & virE & & 1 & pfam05272 & Unique (B5) \\
\hline 529 & holin-like & & 12 & pfam16935 & Accessory (B6, B7); Unique (B5) \\
\hline 555 & PVL (lukF-PV) & & 26 & pfam07968 & Accessory $(B 5, B 6, B 7)$ \\
\hline 914 & $\operatorname{scn}$ & & 17 & pfam11546 & Accessory (B6, B7); Unique (B3) \\
\hline 1259 & pemk & & 10 & pfam02452 & Accessory (B2, B3); Unique (B5) \\
\hline 1270 & virE & & 23 & pfam05272 & Accessory (B5); Unique (B15) \\
\hline 1322 & holin-like & & 1 & pfam16935 & Unique (B6) \\
\hline 1460 & sak & & 16 & pfam02821 & Accessory (B6, B7); Unique (B8) \\
\hline 1579 & $\operatorname{mazF}$ & & 8 & pfam02452 & Accessory (B6) \\
\hline 1597 & $h / b$ & & 1 & Pfam03372 & Unique (B7) \\
\hline 1903 & eta & & 5 & pfam13365 & Accessory (B3); Unique (B2) \\
\hline 1939 & PVL (lukS-PV) & & 27 & pfam07968 & Accessory (B5, B6, B7) \\
\hline 2064 & sea & & 7 & pfam01123; pfam02876 & Accessory (B6) \\
\hline 2122 & chp & & 10 & pfam11434 & Accessory $(B 6, B 7)$ \\
\hline
\end{tabular}

${ }^{a}$ An alternative integrase nomenclature system is provided as in Goerke et al. 2009 (29)

${ }^{b}$ Pham descriptions: pfam14659: Phage integrase, N-terminal SAM-like domain; pfam00589: Phage integrase family; pfam14657: AP2-like DNA-binding integrase domain; pfam00239: Resolvase, N terminal domain; pfam07508: Recombinase; pfam02899: Phage integrase, N-terminal SAM-like domain; pfam13495: Phage integrase, N-terminal SAM-like domain; pfam01123: Staphylococcal/Streptococcal toxin, OB-fold domain; pfam02876: Staphylococcal/Streptococcal toxin, betagrasp domain; pfam02821: Staphylokinase/Streptokinase family; pfam11434: Chemotaxis-inhibiting protein CHIPS; pfam11546: Staphylococcal complement inhibitor SCIN; pfam05272: Virulence-associated protein E; pfam16935: Putative Holin-like Toxin (Hol-Tox); pfam07968: Leukocidin /Hemolysin toxin family; pfam02452: PemK-like, MazF-like toxin of type II toxin-antitoxin system; pfam13365: Trypsin-like peptidase domain; pfam03372:

Endonuclease/Exonuclease/phosphatase family

Acronyms of integrase and virulence genes: Y-Int and S-Int, integrase of tyrosine or serine type; virE, virulence-associated protein E; PVL, Panton-Valentine leucocidin, that is activated by two polypeptide-enconding genes (lukS-PV, lukF-PV); scn, staphylococcal complement inhibitor; pemK, endoribonuclease toxin PemK; sak, plasminogen activator staphylokinase; mazF, endoribonuclease toxin MazF; hlb, $\beta$-hemolysin; eta, exfoliative toxin $A$; sea, staphylococcal enterotoxin $A$; chp, chemotaxis inhibitory protein

Note: The holin-toxin gene is different from the holin gene that participates in the lytic cassette. For instance, in phage P954, gp20 is the holin-toxin, gp21 is the holing and gp22 is the endolysin

from a different pham S. aureus phage TEM126 contains two predicted integrases, one of each catalytic type, a feature also found in Gordonia phages [10]. The roles of the two integrases is unclear. At least five distinct bacterial attachment site (attB) sequences, overlapping host tRNA, tmRNA, lipase $(\mathrm{geh})$ and $\beta$-hemolysin $($ hlb $)$ genes are predicted for phages carrying tyrosine integrase genes (Additional file 34). Collectively, staphylococcal phages exhibit a variety and uncommon number of different site-specific recombinases, like previously observed in Gordonia-infecting phages [10].

\section{Virulence genes are exclusively encoded by cluster B phages}

Staphylococcus prophages have been implicated in the virulence of their hosts through both positive lysogenic conversion, in which prophages encode and express virulence determinants, and through negative lysogenic conversion, in which prophage integration disrupts expression of host encoded virulence associated genes [30]. Prophage interruption of the host $\beta$-hemolysin genes (e.g. phi13 and 42E) or lipase (e.g. phiNM4 and IME1346_01) are associated with $S$. aureus virulence 
$[28,29,31]$. Examples of known prophage-encoded and expressed virulence determinants include the immunemodulator proteins staphylokinase (sak), chemotaxis inhibitory protein of S. aureus (chp) and staphylococcal inhibitor of complement (scn) [28], as well as enterotoxin (sea), exfoliative (eta) and Panton-Valentine leukocidin (lukF-PV or lukS-PV) toxins (reviewed in [30]).

In our dataset we found that Cluster B phages $(n=132)$ have 14 phams associated with virulence determinant functions (Table 1). Interestingly, the genomic location of almost all virulence determinants is between the cell lysis module and the integrase genes (Fig. 3). The identified phams include the above-mentioned sak (pham 1460), chp (pham 2122), scn (pham 914), eta (pham 1903), sea (pham 2064) and lukF-PV or lukS-PV (phams 555 and 1939, respectively), but also the virE (pham 297), holin-like (pham 529), pemK (pham 1259), mazF (pham 1579) and hlb (pham 1597) toxin genes, whose domains associated with virulence determinant functions are described in Table 1 . We note that virulence determinants are highly prevalent and exclusively found in Cluster B phages, although not homogenously distributed throughout the members (Additional file 34). For instance, only 55\% of Cluster B phages have identifiable virulence determinants. There are subclusters that don't have identifiable virulence determinants (B1, B4, B9, B10, B12, B13, B14, B16, and B17), while others have a small (B2 with $32 \%$ and B3 with $31 \%$ ) or a high (B5 with 96\%, B6 and B7 with 100\%) percentage of phages with virulence determinants and often found in every member (Additional file 34). Phages can either encode one (18\%, e.g. SP6), two (14\%, e.g. B236), three (14\%, e.g. LH1), four (8\%, e.g. phiSa119), or even five different virulence determinants $(2 \%$, only found in phiN315 and 3 AJ-2017). Based on our Phamerator dataset, only few virulence determinants could be linked to a specific integrase (e.g. phages with Y-int of pham 148 carrying chp and and interrupting $h l b$ ), however, some were subcluster specific; as virE (pham 1270) and sea (pham 2064) toxins that are associated with subcluster B5 and B6, respectively (Additional file 34). However, we note that genes of unknown function are present in equivalent genome locations in many of the Cluster B phages, and these may represent yet-to-be characterized genes with virulence determinants. Overall, the data obtained reflects the high mosaicism revealed in staphylococci phage genomes and implies a central role of prophages in the evolution and virulence of bacterial pathogens.

\section{Endolysin genes are organized by different strategies}

Phage replication requires a system for progeny release and dispersion to enable new rounds of infection. Multiple strategies, including holin-dependent and holin-independent export to accomplish lysis have been described [32]. The former, typified by phage lambda, is common in dsDNA phages with only some exceptions $[33,34]$. In our analysis, we found 12 different holin and 14 different endolysin phams, perhaps having evolved for effective lysis of diverse staphylococcal strains (Additional file 2).

We have identified four distinct organization strategies of endolysin genes in the staphylococcal phages (Fig. 4a). From a total of 205 Staphylococcus phages, 175 encode endolysins as single genes (e.g. phages 53 and 69), 20 contain group I introns (e.g. phages 85, G1), nine encode endolysins as two adjacent open reading frames (e.g. phages P108, SA11), and one is encoded as a single gene with inter-lytic-domain secondary translation site (phage 2638A) (Additional file 35). The latter endolysin is a unique protein in staphylococcal phage genomes, reported to be expressed as either a endolysin with three lytic domains (a N-terminal peptidase, a centrally located amidase and a C-terminal cell wall binding domain) or a truncated version with only two lytic domains (a $\mathrm{N}$-terminal amidase and a C-terminal cell wall binding domain) [35]. The group I introns have been found in other Staphylococcus phages proteins related to morphogenetic and DNA replication, allowing genome recombination and HGT [36]. Of particular interest is also the evolutionary reason behind phages encoding endolysins in two adjacent genes, which suggests that they might act in a cooperative manner to cleave multiple peptidoglycan bonds. Overall, we did not find any particular pattern between the endolysin organization strategy and phage cluster, morphology or host genus.

To understand the molecular and biological basis of these endolysins, we also analysed the presence of functional domains that bind and degrade the staphylococcal peptidoglycan structure (Fig. 4b). Endolysins may contain several catalytic domains responsible for degradation of different bacterial peptidoglycan substrates, the rarest of these being the PET-M23 (peptidase domain M23) and the most frequent the AMI-2 (amidase 2 domain), AMI-3 (amidase 3 domain) and CHAP (cysteineand histidine-dependent amidohydrolase/peptidase, present in $96 \%$ of the endolysins) (Additional file 35). Conversely, there is only one binding domain belonging to the $\mathrm{SH} 3$ family of proteins identified in $\sim 96 \%$ of the endolysins, that recognises the peptidoglycan substrate, demonstrating a conserved recognition strategy of endolysins to the staphylococcal peptidoglycan polymer.

\section{Discussion}

We describe here the comparative genomic analysis of 205 staphylococci phages isolated at different times and from different geographical regions. S. aureus phages have been previously classified based on morphology and serology [37], or comparative genomics [38, 39]. In 2012, shared gene content based on BLASTP alignments 

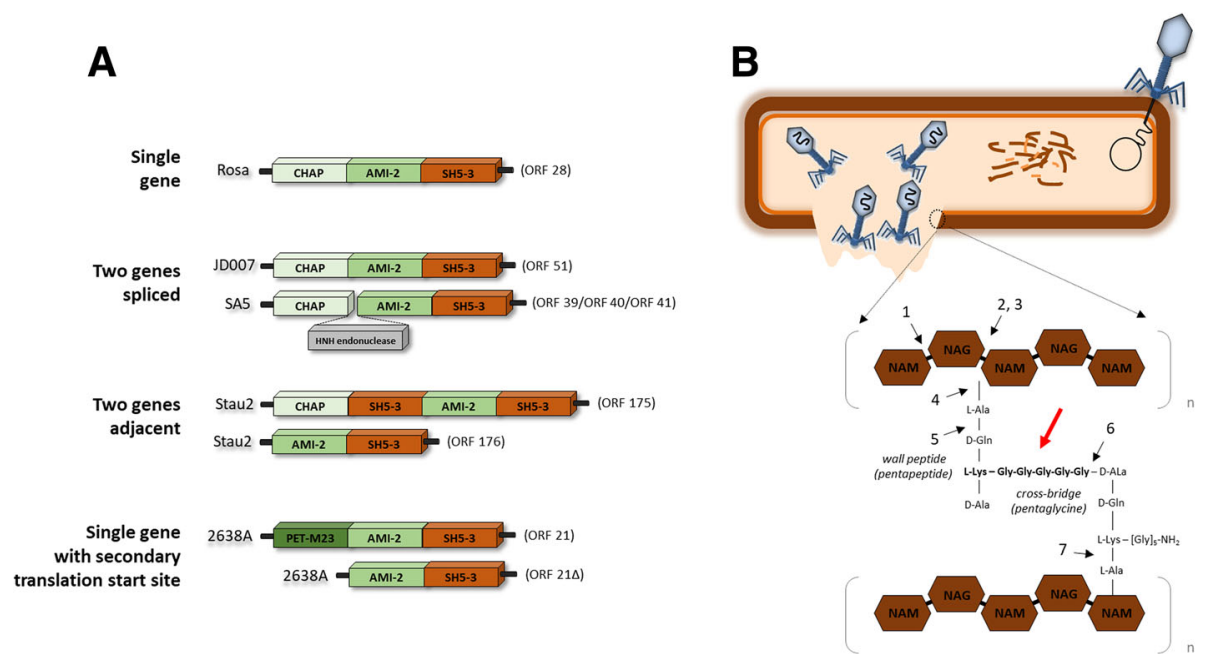

Fig. 4 Organization strategies of staphylococcal phage endolysins. a) Four endolysin synthesis strategies found in staphylococcal phage genomes $(n=205), 175$ phages encode lysins with single genes, 20 phage lysin genes exhibit group I introns, nine phages encode lysins using two adjacent genes; one phage, 2638A, uses a single gene with an inter-lytic-domain secondary translational start site, described here [35]; b) Schematic representation of a $S$. aureus bacteria cell wall and peptidoglycan. The red arrow indicates the conserved glycine peptidoglycan bridge recognized by the SH3 binding domain. Black arrows indicate possible cutting sites of the catalytic domains: 1) N-acetyl- $\beta$-D-glucosaminidase, 2) $\mathrm{N}$-acetyl- $\beta$-D-muramidase, 3) lytic transglycosylase, 4) $\mathrm{N}$-acetylmuramoyl- $\mathrm{L}$-alanine amidase, 5) $\mathrm{L}$-alanyl-D-glutamate endopeptidase, 6) $\mathrm{D}$ -alanyl-glycyl endopeptidase, 7) L-alanoyl-D-glutamate endopeptidase. Endolysins of class 4) and 6) have been experimental validated [60]. Acronyms: CHAP, cysteine/histidine-dependent amidohydrolase/peptidase domain (PF05257/IPR007921); AMI-2, Amidase_2 domain (PF01510/ IPR002502); AMI-3, Amidase_3 domain (PF01520/IPR002508); SH3-5, SH3_5 domain (PF08460/IPR013667); NAG, $\beta$-1,4-linked monomers of N-acetyl glucosamine; NAM; N-acetyl muramic acid

and reciprocal shared matches was used to compute a distance matrix of mostly $S$. aureus-infecting phages $(n$ $=85$ ), revealing three distinct groups matching different morphologies: class I (podoviruses), class II (siphoviruses) and class III (myoviruses), class II was further divided into nine subclusters [19]. In this study, we updated and revised this classification with an additional dataset of genomes $(n=205)$ and including phages infecting other species of Staphylococcus genus. Major contributions to our updated dataset came from phages infecting coagulase-negative staphylococci, which were limited to three phages in the previous analysis (Additional file 1). Following the same division, we have also found three clusters (A, B and $\mathrm{C}$ that correspond to class I, II and III, respectively) but resolved one additional Cluster D (6ec and vB_SepS_SEP9) delineating a group of distinct siphoviruses as well as the singleton (SPbeta-like) (Fig. 1). We also provide a higher resolution of the genetic diversity by subdividing clusters $\mathrm{A}$ and B into several new subclusters that emerged by reorganizing of old and recently deposited phages (Additional file 1 , Additional files $6,7,8,9,10,11,12,13,14$, $15,16,17,18,19,20,21,22,23,24,25,26,27,28,29,30$, $31,32,33)$. The low proportion of singletons found in our phage dataset ( $\mathrm{n}=205,0.5 \%$ are singletons), contrasts with higher numbers found in phages infecting hosts of similar taxonomic level, such as Bacillus ( $n=83,18.1 \%$ are singletons) [14] and Gordonia phages $(n=79,17.7 \%$ are singletons) [10], indicating that the analysed staphylococci phages represent fewer phage types, possibly due to restrictive sampling conditions and methods used. Nonetheless, we show that there is high genetic diversity of staphylococci phage genomes leading to the numerous cluster/subclusters resolved and ORF phamilies obtained ( $n=2139$, where the largest pham has only 101 members) (Additional file 2). Most important would be the isolation of the undersampled coagulase-negative-infecting viruses to reveal the presence of new genes and relationships that shape the evolution of staphylococci phage genomes [40].

Like the Actinobacteriophages, [13, 41, 42], Staphylococcus phage genomes are mosaic in nature, due to horizontal exchange, deletion and addition of ORFs and ORF modules between members (e.g. Additional file 13), likely driven by non-homologous recombination including transposases [43], endonucleases [44] and site-specific recombinases [45] (Additional file 2). Analysis of shared gene content for this dataset via the MaxGCDGap metric $(\sim 0.33$, Fig. $2 d)$ that show low levels of discontinuity across of the spectrum of possible phage genetic relationships, also hints at the active participation of phages in HGT. Moreover, the high number and diversity of phams $(\mathrm{n}=2139$, of which 745 are orphams and without conserved domains) (Additional file 2), also suggest a large influx of genes across novel bacterial hosts and/or other phages by HGT, similar to what was observed in Pseudomonas phages [42]. 
The genetic relationships of the phages described here reflect a general model for phage evolution in which host-virus dynamics have driven diversity over a long period of evolutionary time. Although we have described many distinct lineages with low levels of nucleotide sequence similarity, they have not evolved completely independently, and there are many incidents of horizontal genetic exchange between lineages. These are observed as homologues - albeit distantly related - residing in distinct genomic context in different genomes. Such HGT events are likely to occur when the phages - or their ancestors - are present in the same host species, but the constant pressure to avoid resistance by switching to different hosts confers an impediment to HGT and the emergence of distinct lineages.

Similar to Actinobacteria phages [10], staphylococci Cluster B phages display a large array of lysogenic modules, including multiple integrases, and likely use a variety of attP sites (Table 1 and Additional file 34); a temperate lifestyle has been experimentally demonstrated for several members (e.g. phiNM3, phiNM4, vB_SepiS-phiIPLA5 and vB_SepiS-philPLA7) [21, 46, 47]. Many of these phages are associated with the presence of virulence determinants, which provides the bacterial hosts with additional genes and competitive advantages. For instance, the PVL-carrying phage phiSLT (toxin that causes leukocytolysis and tissues necroris) that lysogenize several PVL-negative $S$. aureus strains using the same 29-bp attB, that subsequently acquire the ability to express the PVL toxin, is an example of prophage contribution for the dissemination and evolution of pathogenicity in staphylococci [47]. The common location of virulence determinants downstream of lysis module (Fig. 3) suggests that expression is coupled to phage induction, a scenario that was experimentally validated for phi13 and other PLV toxin-carrying phages $[48,49]$; however, some virulence determinants may also be expressed from the prophage, as observed for Cluster $\mathrm{N}$ temperate mycobacteriophages [50]. In contrast, some phages have the ability to silence several host proteins associated with virulence determinant functions via disruption of these genes through integration during lysogenization, as it is demonstrated by phage phi13 and the $5^{\prime}$-end of the $\beta$-hemolysin gene (51). However, this loss is usually accompanied with acquisition of new virulence determinants in the staphylococcal chromosome. Thus, by characterizing the current set of staphylococci phage genomes available at the GenBank, we demonstrate a high diversity of integrases and reported novel virulence-associated genes (e.g. hbl and virE) [29], showing a potential for more a versatile role of phages in the adaptive evolution of staphylococcal bacterial pathogens. However, because we have limited our dataset to the complete phage genomes deposited at GenBank at the time of writing, there is a still the potential for additional diverse prophage-derived integrases/virulence determinant genes to be found in staphylococcal genomes. A future similar analysis may identify additional virulence determinants, particularly in phages related to those underrepresented in our current study.

Staphylococci phage endolysin genes are organized in four different manners (single gene, two genes spliced, two genes adjacent and single gene with inter-lytic-domain secondary translational start site), which results in a predicted unusual complex expression system observed in phage genomes (Fig. 4 and Additional file 35). The endolysin functional domains include several catalytic domains but share a single $\mathrm{SH} 3$ binding domain (Additional file 35). It has been proposed that staphylococcal phage endolysin SH3 binds to the peptidoglycan pentaglycine cross-bridge [51]. Lysostaphin (a bacteriocin from Staphylococcus simulans bv. staphylolyticus) and its homologue, hydrolase ALE-1, also display a SH3 domain that recognizes the same epitope $(69,70)$. Therefore, we speculate that SH3 domains of Staphylococcus phage endolysins similarly target glycine-rich bridges identified in 11 out of 12 staphylococci species represented in our study. Despite the conservation of the binding domain, not all of these endolysins are likely to degrade a broad spectrum of staphylococci when added exogenously to liquid cultures, as is observed for endolysins of phages SAP-2 and K, [52, 53], as the endolysin of vB_SauM-LM12 endolysin is specific to $S$. aureus [54]. Additional characterization of the endolysins is required to determine the specificity of the catalytic domains with respect to host strain [54]. Overall, the maintenance of the $\mathrm{SH} 3$ domain despite the various endolysin modular organizations, is a likely response to environmental pressures.

\section{Conclusions}

In summary, by offering a high-resolution and updated view of the staphylococcal viral genetic diversity as well as gene flux patterns within and across different phage groups (cluster and subclusters) we provide novel insights into their evolution. Future biotechnological applications include development of integration-dependent vectors for construction of recombinant staphylococcal strains; and genetic engineering of endolysins for both detection and control of staphylococcal bacterial pathogens.

\section{Methods}

\section{Virus metadata collection}

Biopython 32 package was used within the conda environment (https://www.continuum.io) to retrieve fully sequenced Staphylococcus phage genomes deposited at GenBank as of June $2018(n=205)$ and to create the FASTA files. Python functions scripts were also used to 
collect and list in a table the important features, such as host taxonomy, genome size, GC content, number of ORFs, number of tRNAs, viral taxonomy for each RefSeq record. All metadata retrieved is given in the Additional file 1. Python code created is accessible upon request. During our analysis we excluded the following phages: PT1028 (NC_007045.1), SA1 (NC_027991.1), SpaA1 (NC_018277.1), HOB 14.1.R1 (CP018841.1), pSco-10 (KX011028.1), IME1367_02 (KY653121.1), IME1364_01 (KY653128.1) and SA7 (KY695153.1), for being incomplete or wrongly deposited as Staphylococcus-infecting viruses. Due to strong genomic evidence, we corrected phage UPMK_2 morphology from Podoviridae to Siphoviridae.

\section{Average nucleotide identity values metric}

Average Nucleotide Identity (ANI) values from whole-genome pairwise comparisons were generated with Kalign [55]. Heat map was created in Excel. This was used as the first of a total three metrics used to characterized phage genomes.

\section{Shared gene content metric}

All staphylococcal phage genomes were analysed with Phamerator to group genes into phams - gene products of related proteins [56]. The number of phams generated was used as a second metric to determine phage grouping. First, a SQL database was created locally and customized for incorporation of information present in GenBank files. Second, phage genomes were imported into Phamerator to assign phams with kclust. Phamerator identifies conserved domains in all genes using the NCBI conserved domain database [57]. Analysis were performed on Intel-based PCs with the Windows 7 operating system with a Virtual Machine (Oracle VM Virtual Box) running the Ubuntu 16.04 operating system for execution of Phamerator python scripts in Linux command line. Data manipulation and adjustments in the database scheme were made with MySQL language queries. All the python code created is accessible upon request.

SplitsTree network was used to visualize the relationship of shared gene content between staphylococcal phage genomes $[58,59]$. Phams generated by Phamerator were scored by the presence/absence. Protein repertoire relatedness was used in SplitsTree to visualize and analysed the evolutionary data, using network functionality.

\section{Gene content dissimilarity metric}

The gene content dissimilarities (GCDs) metric was used to further explored phage relationships $[10,11]$. GCD was computed for each pairwise comparison to calculate the number of shared phams between the two divided by the total number of phams present in each genome. The two proportions were also averaged and converted to a gene content dissimilarity: where $\mathrm{GCD}=1$ means $100 \%$ dissimilar (no shared phams) and $\mathrm{GCD}=0$ complete similar (all shared phams). Plots were generated using GCD versus number of pairwise comparisons. The GCD formula is:

$\mathrm{GCD}=1-\left(\frac{\frac{\text { Shared phams }}{\text { Total phams in genome A }}+\frac{\text { Shared phams }}{\text { Total phams in genome B }}}{2}\right)$

Phage-specific MaxGCDGap distances were calculated as previously described [10]. For each phage, all pairwise GCD values were ranked by magnitude, and the difference between each consecutive GCD value was calculated (GCD gap). GCD gap is defined the following formula: $\operatorname{GCDgap}_{(n, n+1)}=\operatorname{GCD}_{n}-G_{C D}+1$. Gap ranges from near 0 (indicating small gene content discontinuities) to 1 (indicating large gene content discontinuities). The MaxGCDGap is the largest of these values. Plots were generated using MaxGCDGap versus pairwise comparisons ordered by magnitude. All GCD related data was calculated with custom written python scripts [11].

\section{Cluster assignment}

Cluster assignment was based on shared gene content. A cut-off of $35 \%$ of shared genes (phams) was used to place phages solely in one cluster, a metric recently used to assigned Gordonia phages [10].

\section{Additional files}

Additional file 1: Staphylococcal phage genome characteristics. Biopython package was used to retrieve complete genome sequences at June of 2018 from NCBI and list in a table with several features associated to the phage. ${ }^{*}$ Numbers in parentheses indicate the size of DNA regions (in bp) homologous to regions of known terminal repeats. DTR, direct terminal repeat; COS, cohesive end site. CoPS, coagulasepositive species, Covs, coagulase-variable species, CoNS, coagulasenegative species. (XLSX $29 \mathrm{~kb}$ )

Additional file 2: Phams. The dataset includes 205 staphylococcal phages, encoding 20,579 predicted ORFs, a total of 2139 phams (gene with related sequences) of which 745 orphams (genes without related sequences) were identified generated based on kclust alignments performed in Phamerator. Phams are ordered by highest conservation among phage members. The phage-ORF column reflects the positioning of genes locus of the genome map generated by Phamerator. Biopython func tions were used to retrieved and list in a table with several features associated to the phages. ${ }^{*}$ An alternative nomenclature system for Cluster B phage integrases is provided as in Goerke et al. 2009 (29). (XLSX 216 kb)

Additional file 3: Average nucleotide sequence identity values. Average nucleotide sequence identities (ANIs) of 205 staphylococcal phage genomes were made using Kalign algorithm. Heat map was created in excel. (XLSX $41 \mathrm{~kb}$ )

Additional file 4: Shared gene content. Biopython functions were used to assignment gene content similarity using the Phamerator output (2139 phams, of which 745 are orphams). Heat map was created in excel. (XLSX 223 kb) 
Additional file 5: Conserved, accessory and unique phams assigned to each subcluster. The distribution of a) Cluster A, b) Cluster B, c) Cluster C and d) Cluster D proteins. Conserved phams are conserved among all members (back). Accessory phams are shared by at least two members (grey). Unique phams are singletons (white). Subclusters B7, B9, B11-B12, B14-B17, C4 and D2 and singleton (SPbeta-like) represented by one member are not shown. Subclusters $A 2, B 13, C 5-C 6$ and D1 represented by two members have no accessory proteins. While there conserved phams among the subclusters can be directly visualized here, phams shared by different groups can be consulted in Additional file 2. As shown in Venn Diagram provided in Fig. 1, there is no universal pham in the staphylococci phage genomes. (PDF $567 \mathrm{~kb}$ )

Additional file 6: Whole-genome map of subcluster A1 phages. Maps were generated using Phamerator in which pairwise sequence similarity (minimal BLASTN cut-off $E$ value is $10^{-4}$ ) is given according to colour spectrum (purple and red lines denote regions of highest and lowest nucleotide similarity, respectively). Ruler corresponds to genome base pairs. Proteins are labelled with predicted function and given a specific colour (shared phams i.e. gene members have the same colour, orphams i.e. unique genes are shown in white). Gene numbering reflects the re-organization of genomes give here to start with packaging genes or at defined ends (all gene related information can be consulted in Additional file 2), and their positioning above or below the bar correspond to rightwards or leftwards transcription, respectively. (PDF $61 \mathrm{~kb}$ )

Additional file 7: Whole-genome map of subcluster A2 phages. Represented as mentioned above. (PDF $20 \mathrm{~kb}$ )

Additional file 8: Whole-genome maps of subcluster B1 phages. Represented as mentioned above. (PDF $87 \mathrm{~kb}$ )

Additional file 9: Whole-genome map of subcluster B2 phages. Represented as mentioned above. (PDF $205 \mathrm{~kb}$ )

Additional file 10: Whole-genome map of subcluster B3 phages. Represented as mentioned above. (PDF $284 \mathrm{~kb}$ )

Additional file 11: Whole-genome map of subcluster B4 phages. Represented as mentioned above. (PDF $111 \mathrm{~kb}$ )

Additional file 12: Whole-genome map of subcluster B5 phages. Represented as mentioned above. (PDF $271 \mathrm{~kb}$ )

Additional file 13: Whole-genome maps of subcluster B6 phages. Represented as mentioned above. (PDF $195 \mathrm{~kb}$ )

Additional file 14: Whole-genome map of subcluster B7 phages. Represented as mentioned above. (PDF $136 \mathrm{~kb}$ )

Additional file 15: Whole-genome map of subcluster B8 phages. Represented as mentioned above. (PDF $37 \mathrm{~kb}$ )

Additional file 16: Whole-genome map of subcluster B9 phages. Represented as mentioned above. (PDF $35 \mathrm{~kb}$ )

Additional file 17: Whole-genome map of subcluster B10 phages. Represented as mentioned above. (PDF $50 \mathrm{~kb}$ )

Additional file 18: Whole-genome map of subcluster B11 phages. Represented as mentioned above. (PDF $23 \mathrm{~kb}$ )

Additional file 19: Whole-genome maps of subcluster B12 phages. Represented as mentioned above. (PDF $24 \mathrm{~kb}$ )

Additional file 20: Whole-genome map of subcluster B13 phages. Represented as mentioned above. (PDF $36 \mathrm{~kb}$ )

Additional file 21: Whole-genome map of subcluster B14 phages. Represented as mentioned above. (PDF $24 \mathrm{~kb}$ )

Additional file 22: Whole-genome map of subcluster B15 phages. Represented as mentioned above. (PDF $15 \mathrm{~kb}$ )

Additional file 23: Whole-genome map of subcluster B16 phages. Represented as mentioned above. (PDF $23 \mathrm{~kb}$ )

Additional file 24: Whole-genome maps of subcluster B17 phages. Represented as mentioned above. (PDF $24 \mathrm{~kb}$ )

Additional file 25: Whole-genome map of subcluster C1 phages. Represented as mentioned above. (PDF $1182 \mathrm{~kb}$ )
Additional file 26: Whole-genome map of subcluster C2 phages. Represented as mentioned above. (PDF $182 \mathrm{~kb}$ )

Additional file 27: Whole-genome map of subcluster C3 phages. Represented as mentioned above. (PDF $170 \mathrm{~kb}$ )

Additional file 28: Whole-genome map of subcluster $C 4$ phages. Represented as mentioned above. (PDF $37 \mathrm{~kb}$ )

Additional file 29: Whole-genome map of subcluster $C 5$ phages. Represented as mentioned above. (PDF $72 \mathrm{~kb}$ )

Additional file 30: Whole-genome map of subcluster $\mathrm{C} 6$ phages. Represented as mentioned above. (PDF $76 \mathrm{~kb}$ )

Additional file 31: Whole-genome map of subcluster D1 phages. Represented as mentioned above. (PDF $55 \mathrm{~kb}$ )

Additional file 32: Whole-genome map of subcluster D2 phages. Represented as mentioned above. (PDF $35 \mathrm{~kb}$ )

Additional file 33: Whole-genome map of subcluster singleton SPbetalike. Represented as mentioned above. (PDF $40 \mathrm{~kb}$ )

Additional file 34: Integrases, attB sites and virulence genes. The dataset includes all cluster B staphylococcal siphoviruses $(n=132)$, for which five integrases types, five attB sites and 13 virulence factors were identified. The integrases were identified through Phamerator and BLASTP. The attB site was found through BLASTP using best host species hit similar to the one originally used to isolate the phage. The virulence genes were retrieved from Additional file 2 and reorganized. (XLSX $72 \mathrm{~kb}$ )

Additional file 35: Endolysin genes. The dataset includes 205 staphylococcal phages, for which four distinct organization strategies were found (single gene, two genes spliced, two genes adjacent and single gene with inter-lytic-domain secondary translational start site). The corresponding nucleotide and amino acid sequences and functional encoding domains (catalytic and binding) detected through the structural database HHpred are given. All genes spliced with group I introns were manually curated. (XLSX $99 \mathrm{~kb}$ )

\section{Abbreviations}

ANI: Average nucleotide identity; attB: Bacterial attachment site; chp: Chemotaxis inhibitory protein; GCD: Gene content dissimilarity; geh: Lipase; HGT: Horizontal gene transfer; hlb: $\beta$-hemolysin; Int: Integrase of tyrosine ( $($-Int) or serine (S-Int) type; lukS-PV and lukF-PV: Panton-Valentine bi-component leukocidin subunits S or F; mazF: Endoribonuclease toxin MazF; sak: Plasminogen activator staphylokinase; sea: Staphylococcal enterotoxin A; snc: Staphylococcal complement inhibitor

\section{Acknowledgments}

We acknowledge Travis Mavrich for the preliminary data processing assistance provided.

\section{Funding}

Financial support was provided by the Foundation for Science and Technology (FCT) under the scope of the strategic funding of UID/BIO/ 04469/2013 unit, COMPETE 2020 (POCl-01-0145-FEDER-006684) and FCT grant FRH/BPD/111653/2015 to HO. Funding was also provided by National Institutes of Health grant GM116884 and Howard Hughes Medical Institute grant 54308198 to GH. These funding bodies had no role in the design of the study, collection, analysis, and interpretation of data, or in writing the manuscript.

\section{Availability of data and materials}

All data generated or analysed during this study are included in this published article and its supplementary information files.

\section{Authors' contributions}

$\mathrm{HO}$ conceived, analysed data, wrote the manuscript and produced tables and Figures MS and OD compiled the staphylococcus phage Phamerator database and wrote the python code. LM analysed and wrote the manuscript. WP, GH and JA conceived the study, analysed and edited the manuscript. All authors approved the final manuscript. 


\section{Ethics approval and consent to participate}

Not applicable.

\section{Consent for publication}

Not applicable.

\section{Competing interests}

The authors declare that they have no competing interests.

\section{Publisher's Note}

Springer Nature remains neutral with regard to jurisdictional claims in published maps and institutional affiliations.

\section{Author details}

'CEB - Centre of Biological Engineering, University of Minho, Braga, Portugal. ${ }^{2}$ Department of Biological Sciences, University of Pittsburgh, Pittsburgh, PA, USA.

Received: 22 January 2019 Accepted: 27 March 2019

Published online: 09 May 2019

\section{References}

1. Edwards RA, Rohwer F. Viral metagenomics. Nat Rev Microbiol. 2005;3(6):504-10.

2. $\quad$ Rohwer F. Global phage diversity. Cell. 2003;113(2):141.

3. Canchaya C, Fournous G, Brussow $\mathrm{H}$. The impact of prophages on bacterial chromosomes. Mol Microbiol. 2004;53(1):9-18.

4. Bondy-Denomy J, Qian J, Westra ER, Buckling A, Guttman DS, Davidson AR, Maxwell KL. Prophages mediate defense against phage infection through diverse mechanisms. ISME J. 2016;10(12):2854-66.

5. Mc Grath S, Fitzgerald GF, van Sinderen D. Bacteriophages in dairy products: pros and cons. Biotechnol J. 2007:2(4):450-5.

6. Wilhelm SW, Suttle CA. Viruses and nutrient cycles in the sea - viruses play critical roles in the structure and function of aquatic food webs. Bioscience. 1999;49(10):781-8.

7. Brussow H, Canchaya C, Hardt WD. Phages and the evolution of bacterial pathogens: from genomic rearrangements to lysogenic conversion. Microbiol Mol Biol R. 2004;68(3):560-+.

8. Canchaya C, Fournous G, Chibani-Chennoufi S, Dillmann ML, Brussow $\mathrm{H}$. Phage as agents of lateral gene transfer. Curr Opin Microbiol. 2003;6(4):417-24.

9. Hendrix RW, Smith MC, Burns RN, Ford ME, Hatfull GF. Evolutionary relationships among diverse bacteriophages and prophages: all the world's a phage. Proc Natl Acad Sci U S A. 1999;96(5):2192-7.

10. Pope WH, Mavrich TN, Garlena RA, Guerrero-Bustamante CA, Jacobs-Sera D, Montgomery MT, Russell DA, Warner MH, Hatfull GF. Bacteriophages of Gordonia spp. display a Spectrum of diversity and genetic relationships. mBio. 2017;8(4).

11. Mavrich TN, Hatfull GF. Bacteriophage evolution differs by host, lifestyle and genome. Nat Microbiol. 2017;2:17112.

12. Pope WH, Bowman CA, Russell DA, Jacobs-Sera D, Asai DJ, Cresawn SG, Jacobs WR, Hendrix RW, Lawrence JG, Hatfull GF. Whole genome comparison of a large collection of mycobacteriophages reveals a continuum of phage genetic diversity. eLife. 2015;4:e06416.

13. Grose JH, Casjens SR. Understanding the enormous diversity of bacteriophages: the tailed phages that infect the bacterial family Enterobacteriaceae. Virology. 2014;468-470:421-43.

14. Grose JH, Jensen GL, Burnett SH, Breakwell DP. Genomic comparison of 93 Bacillus phages reveals 12 clusters, 14 singletons and remarkable diversity. BMC Genomics. 2014;15:855.

15. Klyczek KK, Bonilla JA, Jacobs-Sera D, Adair TL, Afram P, Allen KG, Archambault ML, Aziz RM, Bagnasco FG, Ball SL, et al. Tales of diversity: genomic and morphological characteristics of forty-six Arthrobacter phages. PLoS One. 2017;12(7):e0180517.

16. Pedulla ML, Ford ME, Houtz JM, Karthikeyan T, Wadsworth C, Lewis JA, Jacobs-Sera D, Falbo J, Gross J, Pannunzio NR, et al. Origins of highly mosaic mycobacteriophage genomes. Cell. 2003;113(2):171-82.

17. Hatfull GF, Jacobs-Sera D, Lawrence JG, Pope WH, Russell DA, Ko CC, Weber RJ, Patel MC, Germane KL, Edgar RH, et al. Comparative genomic analysis of 60 Mycobacteriophage genomes: genome clustering, Gene Acquisition, and gene size. J Mol Biol. 2010;397(1):119-43.
18. Hatfull GF, Cresawn SG, Hendrix RW. Comparative genomics of the mycobacteriophages: insights into bacteriophage evolution. Res Microbiol. 2008;159(5):332-39.

19. Deghorain M, Bobay LM, Smeesters PR, Bousbata S, Vermeersch M, PerezMorga D, Dreze PA, Rocha EP, Touchon M, Van Melderen L. Characterization of novel phages isolated in coagulase-negative staphylococci reveals evolutionary relationships with Staphylococcus aureus phages. J Bacteriol. 2012;194(21):5829-39.

20. Cater K, Dandu VS, Bari SM, Lackey K, Everett GF, Hatoum-Aslan A. A Novel Staphylococcus Podophage Encodes a Unique Lysin with Unusual Modular Design. mSphere. 2017;2(2).

21. Bae T, Baba T, Hiramatsu K, Schneewind O. Prophages of Staphylococcus aureus Newman and their contribution to virulence. Mol Microbiol. 2006;62(4):1035-47.

22. Gutierrez D, Martinez B, Rodriguez A, Garcia P. Isolation and characterization of bacteriophages infecting Staphylococcus epidermidis. Curr Microbiol. 2010;61(6):601-8.

23. Zou D, Kaneko J, Narita S, Kamio Y. Prophage, phi PV83-pro, carrying panton-valentine leukocidin genes, on the Staphylococcus aureus p83 chromosome: comparative analysis of the genome structures of phi PV83-pro, phi PVL, phi 11, and other phages. Biosci Biotechnol Biochem. 2000;64(12):2631-43.

24. Smeltzer MS, Hart ME, landolo JJ. The effect of Lysogeny on the genomic organization of Staphylococcus aureus. Gene. 1994;138(1-2):51-7.

25. Lobocka M, Hejnowicz MS, Dabrowski K, Gozdek A, Kosakowski J, Witkowska M, Ulatowska MI, Weber-Dabrowska B, Kwiatek M, Parasion S, et al. Genomics of staphylococcal Twort-like phages--potential therapeutics of the post-antibiotic era. Advances in virus research. 2012;83:143-216.

26. Aswani VH, Tremblay DM, Moineau S, Shukla SK. Complete genome sequence of a Staphylococcus epidermidis bacteriophage isolated from the anterior nares of humans. Genome announcements. 2014;2(4).

27. Melo LD, Sillankorva S, Ackermann HW, Kropinski AM, Azeredo J, Cerca N. Characterization of Staphylococcus epidermidis phage VB_SepS_SEP9 - a unique member of the Siphoviridae family. Res Microbiol. 2014;165(8):679-85.

28. Coleman DC, Sullivan DJ, Russell RJ, Arbuthnott JP, Carey BF, Pomeroy HM. Staphylococcus aureus bacteriophages mediating the simultaneous lysogenic conversion of beta-lysin, staphylokinase and enterotoxin a: molecular mechanism of triple conversion. J Gen Microbiol. 1989;135(6):1679-97.

29. Goerke C, Pantucek R, Holtfreter S, Schulte B, Zink M, Grumann D, Broker BM, Doskar J, Wolz C. Diversity of prophages in dominant Staphylococcus aureus clonal lineages. J Bacteriol. 2009;191(11):3462-8.

30. Malachowa N, DeLeo FR. Mobile genetic elements of Staphylococcus aureus. Cellular and molecular life sciences: CMLS. 2010;67(18):3057-71.

31. landolo JJ, Worrell V, Groicher KH, Qian Y, Tian R, Kenton S, Dorman A, Ji H, Lin $\mathrm{S}$, Loh $\mathrm{P}$, et al. Comparative analysis of the genomes of the temperate bacteriophages phi 11, phi 12 and phi 13 of Staphylococcus aureus 8325. Gene. 2002:289(1-2):109-18.

32. Young R. Phage lysis: three steps, three choices, one outcome. J Microbiol. 2014:52(3):243-58.

33. Sao-Jose C, Parreira R, Vieira G, Santos MA. The N-terminal region of the Oenococcus oeni bacteriophage fOg44 lysin behaves as a bona fide signal peptide in Escherichia coli and as a cis-inhibitory element, preventing lytic activity on oenococcal cells. J Bacteriol. 2000;182(20):5823-31.

34. Kakikawa M, Yokoi K, Kimoto H, Nakano M, Kawasaki K, Taketo A, Kodaira K Molecular analysis of the lysis protein Lys encoded by Lactobacillus plantarum phage phig1e. Gene. 2002;299(1-2):227-34.

35. Abaev I, Foster-Frey J, Korobova O, Shishkova N, Kiseleva N, Kopylov P, Pryamchuk S, Schmelcher M, Becker SC, Donovan DM. Staphylococcal phage 2638A endolysin is lytic for Staphylococcus aureus and harbors an inter-lytic-domain secondary translational start site. Appl Microbiol Biotechnol. 2013:97(8):3449-56.

36. Vandersteegen K, Kropinski AM, Nash JH, Noben JP, Hermans K, Lavigne R Romulus and Remus, two phage isolates representing a distinct clade within the Twortlikevirus genus, display suitable properties for phage therapy applications. J Virol. 2013;87(6):3237-47.

37. Pantucek R, Doskar J, Ruzickova V, Kasparek P, Oracova E, Kvardova V, Rosypal S. Identification of bacteriophage types and their carriage in Staphylococcus aureus. Arch Virol. 2004;149(9):1689-703.

38. Daniel A, Bonnen PE, Fischetti VA. First complete genome sequence of two Staphylococcus epidermidis bacteriophages. J Bacteriol. 2007;189(5):2086-100. 
39. Kwan T, Liu J, DuBow M, Gros P, Pelletier J. The complete genomes and proteomes of 27 Staphylococcus aureus bacteriophages. Proc Natl Acad Sc U S A. 2005;102(14):5174-9.

40. Bray JE, Todd AE, Pearl FM, Thornton JM, Orengo CA. The CATH dictionary of homologous Superfamilies (DHS): a consensus approach for identifying distant structural homologues. Protein Eng. 2000;13(3):153-65.

41. Pope WH, Jacobs-Sera D, Russell DA, Peebles CL, Al-Atrache Z, Alcoser TA, Alexander LM, Alfano MB, Alford ST, Amy NE, et al. Expanding the diversity of mycobacteriophages: insights into genome architecture and evolution. PLoS One. 2011;6(1):e16329.

42. Ha AD, Denver DR. Comparative genomic analysis of 130 bacteriophages infecting Bacteria in the genus Pseudomonas. Front Microbiol. 2018;9:1456.

43. Edgell DR, Gibb EA, Belfort M. Mobile DNA elements in T4 and related phages. Virol J. 2010;7:290.

44. Kristensen DM, Waller AS, Yamada T, Bork P, Mushegian AR, Koonin EV. Orthologous gene clusters and taxon signature genes for viruses of prokaryotes. J Bacteriol. 2013;195(5):941-50.

45. De Paepe M, Hutinet G, Son O, Amarir-Bouhram J, Schbath S, Petit MA. Temperate phages acquire DNA from defective prophages by relaxed homologous recombination: the role of Rad52-like recombinases. PLoS Genet. 2014;10(3):e1004181.

46. Kaneko J, Kimura T, Narita S, Tomita T, Kamio Y. Complete nucleotide sequence and molecular characterization of the temperate staphylococcal bacteriophage phiPVL carrying Panton-valentine leukocidin genes. Gene. 1998;215(1):57-67.

47. Narita S, Kaneko J, Chiba J, Piemont Y, Jarraud S, Etienne J, Kamio Y. Phage conversion of Panton-valentine leukocidin in Staphylococcus aureus: molecular analysis of a PVL-converting phage, phiSLT. Gene. 2001;268(1-2):195-206.

48. Goerke C, Koller J, Wolz C. Ciprofloxacin and trimethoprim cause phage induction and virulence modulation in Staphylococcus aureus. Antimicrob Agents Chemother. 2006;50(1):171-7.

49. Wirtz C, Witte W, Wolz C, Goerke C. Transcription of the phage-encoded Panton-valentine leukocidin of Staphylococcus aureus is dependent on the phage life-cycle and on the host background. Microbiol-Sgm. 2009;155:3491-9.

50. Dedrick RM, Jacobs-Sera D, Bustamante CAG, Garlena RA, Mavrich TN, Pope WH, Reyes JCC, Russell DA, Adair T, Alvey R, et al. Prophage-mediated defence against viral attack and viral counter-defence. Nat Microbiol. 2017;2(3):16251.

51. Benesik M, Novacek J, Janda L, Dopitova R, Pernisova M, Melkova K, Tisakova L, Doskar J, Zidek L, Hejatko J, et al. Role of SH3b binding domain in a natural deletion mutant of Kayvirus endolysin LysF1 with a broad range of lytic activity. Virus Genes. 2018;54(1):130-9.

52. Son JS, Lee SJ, Jun SY, Yoon SJ, Kang SH, Paik HR, Kang JO, Choi YJ. Antibacterial and biofilm removal activity of a podoviridae Staphylococcus aureus bacteriophage SAP-2 and a derived recombinant cell-wall-degrading enzyme. Appl Microbiol Biotechnol. 2010;86(5):1439-49.

53. O'Flaherty S, Coffey A, Meaney W, Fitzgerald GF, Ross RP. The recombinant phage lysin LysK has a broad spectrum of lytic activity against clinically relevant staphylococci, including methicillin-resistant Staphylococcus aureus. J Bacteriol. 2005:187(20):7161-4

54. Melo LDR, Brandao A, Akturk E, Santos SB, Azeredo J. Characterization of a new Staphylococcus aureus Kayvirus harboring a Lysin active against biofilms. Viruses. 2018;10(4)

55. Lassmann T, Sonnhammer EL. Kalign--an accurate and fast multiple sequence alignment algorithm. BMC bioinformatics. 2005;6:298.

56. Cresawn SG, Bogel M, Day N, Jacobs-Sera D, Hendrix RW, Hatfull GF. Phamerator: a bioinformatic tool for comparative bacteriophage genomics. BMC bioinformatics. 2011;12:395.

57. Marchler-Bauer A, Panchenko AR, Shoemaker BA, Thiessen PA, Geer LY, Bryant SH. CDD: a database of conserved domain alignments with links to domain three-dimensional structure. Nucleic Acids Res. 2002;30(1):281-3.

58. Huson DH. SplitsTree: analyzing and visualizing evolutionary data. Bioinformatics. 1998;14(1):68-73.

59. Huson DH, Bryant D. Application of phylogenetic networks in evolutionary studies. Mol Biol Evol. 2006;23(2):254-67.

60. Navarre WW, Ton-That H, Faull KF, Schneewind O. Multiple enzymatic activities of the murein hydrolase from staphylococcal phage phi11. Identification of a D-alanyl-glycine endopeptidase activity. J Biol Chem. 1999;274(22):15847-56

\section{Ready to submit your research? Choose BMC and benefit from:}

- fast, convenient online submission

- thorough peer review by experienced researchers in your field

- rapid publication on acceptance

- support for research data, including large and complex data types

- gold Open Access which fosters wider collaboration and increased citations

- maximum visibility for your research: over $100 \mathrm{M}$ website views per year

At BMC, research is always in progress.

Learn more biomedcentral.com/submissions 